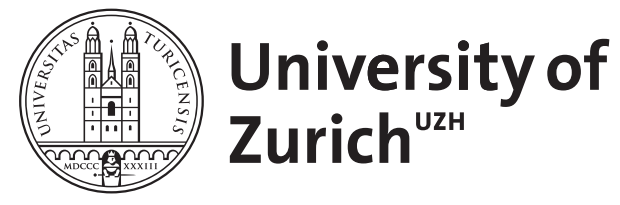

Preventing parastomal hernia with a prosthetic mesh: a five year follow up of a randomised study

Schäfer, Markus

DOI: https://doi.org/10.1007/s00268-008-9803-6

Posted at the Zurich Open Repository and Archive, University of Zurich ZORA URL: https://doi.org/10.5167/uzh-156171

Journal Article

Published Version

Originally published at:

Schäfer, Markus (2009). Preventing parastomal hernia with a prosthetic mesh: a five year follow up of a randomised study. World Journal of Surgery, 33(1):122-123.

DOI: https://doi.org/10.1007/s00268-008-9803-6 


\title{
Preventing Parastomal Hernia with a Prosthetic Mesh: A Five Year Follow Up of a Randomised Study
}

\author{
Markus Schäfer
}

Published online: 21 November 2008

(c) Société Internationale de Chirurgie 2008

Definitive colostomy formation has become less frequent during the last two decades since abdominoperineal resection can now be omitted in many patients with rectal cancer, and Hartmann's operation for sigmoid diverticulitis is increasingly replaced by resection with primary anastomosis-with a loop ileostomy, if necessary. Nevertheless, there are still a number of patients who will receive a definitive colostomy due to different reasons. Long-term quality of life in those patients is significantly correlated to a "good" colostomy that comprises a proper localization in the abdominal wall, a non-impaired evacuation, and finally an easy management of the ostomy devices. While patients with a well functioning colostomy have few restrictions in their daily live, patients with ostomy problems may be heavily disturbed in their individual quality of life. Furthermore, patients with parastomal hernia often need additional ostomy devices, i.e. specially shaped faceplates, and repeated use of health care resources, both causing a significant increase of cost.

Development of parastomal hernia is a common problem that occurs after definitive colostomy creation. The reported incidence ranges up to $50 \%$. Surgical repair is performed in at least $30 \%$ of all patients with parastomal hernia, whereby the postoperative recurrence rate is high. Colostomies are at particular risk to develop parastomal hernia since intraoperatively it is impossible to exactly determine the precise diameter of the hole; in reality it is always a compromise between keeping the opening narrow versus not impairing blood flow as well as stool evacuation. The passage of the colon through the abdominal wall

M. Schäfer $(\square)$

Department of Visceral and Transplantation Surgery, University Hospital of Zurich, Zurich, Switzerland

e-mail: markus.schaefer@usz.ch represents therefore a kind of a "planned" weakness heralding hernia formation. This is in contrast to "normal" incisions of the abdominal wall that are tightly closed after the intervention.

The high incidence of parastomal hernia with its subsequent adverse effects on quality of life, the need for surgical repair, and increased cost provide the impetus to prevent parastomal hernia formation instead of just repairing the defect. In accordance to the convincing results of incisional hernia repair by using a prosthetic mesh, it seems logical to insert a prosthetic mesh during the index operation in order to obviate parastomal hernia formation. But traditionally prosthetic meshes have only be used in clean, not contaminated or infectious situations, since infections of prosthetic material has been feared as major complication. However, new mesh types have been developed during recent years and are currently available. For example, light weight meshes with partially absorbable components and coated meshes for intraabdominal use revealed both a high clinical safety, hence permitting a broader use.

The study by Jänes et al. [1] reports the 5-years followup of a randomized trial comparing prophylactic mesh implantation versus colostomy formation without using a mesh. So far, this is the only randomized trial that addresses this important topic. Light weight meshes with partially absorbable components that are placed in a sublay position provided impressively low rates of parastomal hernia. The results clearly showed a benefit for the mesh group (7.5\% versus $63 \%$ parastomal hernia formation). In this study, parastomal hernia formation was an ongoing process, showing an increasing incidence over the years. Furthermore, the rate of stricture and fistula formation was low. Similar results were reported in some other non-randomized series. 
Based on the current evidence, prophylactic implantation of a mesh should probably be advised for all patients. But there remain some important questions: How should a prosthetic mesh be used in patients undergoing laparoscopic resections? Which is the best mesh? What is the optimal diameter of the opening in the mesh? What is the best position of the mesh? Can a mesh safely be used during emergency operation with a severe contamination of the abdominal cavity?

\section{References}

1. Janes A, Israelsson L, Cengiz Y, Preventing Parastomal Hernia with a Prosthetic Mesh: a five year follow up of a Randomized Study. World J Surg. doi:10.1007/s00268-008-9785-4 [Epub ahead of print] 